ournal for ImmunoTherapy of Cancer

\section{Immune adverse events (irAEs) with adjuvant ipilimumab in melanoma, use of immunosuppressants and association with outcome: ECOG-ACRIN E1609 study analysis}

To cite: Tarhini AA, Kang N, Lee SJ, et al. Immune adverse events (irAEs) with adjuvant ipilimumab in melanoma, use of immunosuppressants and association with outcome: ECOG-ACRIN E1609 study analysis. Journal for ImmunoTherapy of Cancer 2021;9:e002535. doi:10.1136/ jitc-2021-002535

- Additional supplemental material is published online only. To view, please visit the journal online (http://dx.doi.org/10. 1136/jitc-2021-002535).

Accepted 27 March 2021
Check for updates

(C) Author(s) (or their employer(s)) 2021. Re-use permitted under CC BY-NC. No commercial re-use. See rights and permissions. Published by BMJ.

For numbered affiliations see end of article.

Correspondence to

Dr Ahmad A Tarhini;

ahmad.tarhini@moffitt.org

\section{ABSTRACT}

Background The impact of immune-related adverse events (irAEs) occurring from adjuvant use of immunotherapy and of their management on relapsefree survival (RFS) and overall survival (OS) outcomes is currently not well understood.

Patients and methods E1609 enrolled 1673 patients with resected high-risk melanoma and evaluated adjuvant ipilimumab $3 \mathrm{mg} / \mathrm{kg}$ (ipi3) and $10 \mathrm{mg} / \mathrm{kg}$ (ipi10) versus interferon- $\alpha$. We investigated the association of irAEs and of use of immunosuppressants with RFS and OS for patients treated with ipilimumab $(n=1034)$.

Results Occurrence of grades 1-2 irAEs was associated with RFS (5 years: $52 \%$ ( $95 \%$ Cl $47 \%$ to $56 \%$ ) vs $41 \%$ (95\% $\mathrm{Cl} 31 \%$ to $50 \%$ ) with no $\mathrm{AE} ; \mathrm{p}=0.006$ ) and a trend toward improved 0S (5 years: $75 \%$ (95\% Cl $71 \%$ to $79 \%$ ) compared with $67 \%$ (95\% Cl $56 \%$ to $75 \%$ ) with no $\mathrm{AE}$; $\mathrm{p}=0.064)$. Among specific irAEs, grades $1-2$ rash was most significantly associated with RFS $(p=0.002)$ and $0 S$ $(p=0.003)$. In multivariate models adjusting for prognostic factors, the most significant associations were seen for grades $1-2$ rash with RFS $(p<0.001, H R=0.70)$ and $0 S$ $(p=0.01, H R=0.71)$ and for grades $1-2$ endocrine+rash with RFS $(p<0.001, H R=0.66)$ and $0 S(p=0.008, H R=0.7)$. Overall, grades 1-2 irAEs had the best prognosis in terms of RFS and OS and those with grades 3-4 had less RFS benefits and no $0 \mathrm{~S}$ advantage over no irAE. Patients experiencing grades 3-4 irAE had significantly higher exposure to corticosteroids and immunosuppressants than those with grades $1-2$ (92\% vs $60 \% ; p<0.001)$, but no significant associations were found between corticosteroid and immunosuppressant use and RFS or OS. In investigating the impact of non-corticosteroid immunosuppressants, although there were trends toward better RFS and OS favoring cases who were not exposed, no significant associations were found.

Conclusions Rash and endocrine irAEs were independent prognostic factors of RFS and OS in patients treated with adjuvant ipilimumab. Patients experiencing lower grade irAEs derived the most benefit, but we found no significant evidence supporting a negative impact of high dose corticosteroids and immunosuppressants more commonly used to manage grades 3-4 irAEs.

\section{INTRODUCTION}

Immune-related adverse events (irAEs) associated with immune checkpoint inhibitor (ICI) therapy represent a new and major challenge to patients and managing clinicians. ${ }^{12}$ The incidence of irAEs varies by the type of ICI used and is lowest with anti-PD1/PDL1 antibodies, followed by anti-CTLA4 and is highest with the combination of anti-PD1 and anti-CTLA4 antibodies. ${ }^{2}$ CTLA-4 and $\mathrm{PD}-1$ receptors have distinct mechanisms in $\mathrm{T}$ cell differentiation and function. While antiCTLA-4 antibodies release $\mathrm{T}$ cell inhibition at the priming phase of the antitumor immune response, anti-PD-1 antibodies release $\mathrm{T}$ cell inhibition at the effector phase. Furthermore, dual blockade of CTLA-4 and PD-1 appears to lead to a potentially synergistic or additive effect, not only in terms of antitumor activity but also as related to the risk of irAEs. ${ }^{3}$ The incidence of irAEs following CTLA4 blockade is dose dependent and increases with the dose of ICI used where our main experience is derived from studies that tested ipilimumab. In the CA184-169 study that tested ipilimumab at $3 \mathrm{mg} / \mathrm{kg}$ (ipi3) and ipilimumab at 10 $\mathrm{mg} / \mathrm{kg}$ (ipi10) in metastatic melanoma, the incidence of grades 3-4 irAEs was $14 \%$ and $30 \%$, respectively. ${ }^{4}$ Similarly, the same dose levels tested as adjuvant therapy for high-risk resected melanoma in the E1609 trial led to an incidence of $19 \%$ and $34 \%$ of grades $3-4$ 
irAEs, respectively. ${ }^{5}$ More importantly, irAEs can lead to treatment discontinuation, can be life threatening and rarely lead to death. Management of irAEs can also be complex and frequently requires the use corticosteroids, immunosuppressants and hormone replacement therapy. In the context of E1609, the toxicity attrition rate related to irAEs was $35 \%$ with ipi3 and $54 \%$ with ipi $10 .{ }^{5}$ Therefore, there is a need to better understand irAEs and their management strategies and their association with clinical outcomes.

North American Intergroup trial E1609 tested ipi3 and ipi10 as adjuvant therapy as compared with highdose interferon- $\alpha$ (HDI) and demonstrated significant improvement in overall survival (OS) with ipi3 compared with HDI and less toxicity with ipi3 compared with ipi $10 .{ }^{6}$ This trial was the largest adjuvant trial reported to date in melanoma, with participation from 1673 patients including 1034 patients treated with ipilimumab. ${ }^{6}$ This trial was unique in investigating varying dose levels with an ICI in the melanoma phase 3 adjuvant setting through an international consortium of sites across the USA and Canada. The study was conducted at a time when relatively little experience with immune checkpoint blockade was present among treating oncologists. Therefore, it provided for continuous and close monitoring of irAEs and offered unprecedented comprehensive educational and training efforts in the management of irAEs. In this report, we provide the outcome of our in-depth analysis of irAEs with ipi3 and ipi10 in E1609 and the use of immunosuppressants and hormone replacement therapy and the association with outcome.

\section{PATIENTS AND METHODS Patients}

E1609 enrolled patients with melanoma of cutaneous or unknown primary origin that was histologically confirmed. Patients were required to have American Joint Committee on Cancer (AJCC) 7th edition stages IIIB, IIIC, M1a or $\mathrm{M} 1 \mathrm{~b}$ and were rendered disease free surgically within 12 weeks of randomization on the trial. ${ }^{6}$ An Eastern Cooperative Oncology Group (ECOG) performance status (PS) of 0 or 1 and screening safety laboratory test criteria were mandated. No history of autoimmune disorders or conditions of immunosuppression that necessitated the use of systemic corticosteroids or other immunosuppressants was allowed.

\section{Trial design and treatments}

E1609 was an open-label phase III trial that randomized patients to adjuvant therapy with either ipi3, HDI or ipi10. Patients were stratified by the AJCC 7 th edition stage (IIIB, IIIC, Mla and M1b) ${ }^{6}$ Details of the treatment regimens, randomization, clinical trial endpoint points and trial oversight were previously published. ${ }^{6}$

\section{Methods and statistical analysis}

IrAEs were defined as select adverse events (AEs) of interest that were considered to be consistent with ICIs. ${ }^{1}$
Descriptive statistics were used to evaluate the distributions of irAEs by type and grade, and the use of corticosteroids, immunosuppressants and hormone replacement therapy on the ipi arms. For each irAE type, the worst degree was assessed from each patient. Then the worst degree was categorized as grades 1-2 or grades 1-4. Individuals with no irAE of the type under study was considered as no irAEs. For multiple irAEs, the worst degree was assessed among the specified multiple irAE types. Patients with no irAEs of any of the multiple irAE were coded as no irAE. Patients with grade 5 AEs were not included.

The association between the use of corticosteroids and immunosuppressants and clinical outcome was tested. Distributions of OS and relapse-free survival (RFS) were estimated using the Kaplan-Meier method by irAE type/ grade and compared using the log-rank test. ${ }^{7}$ Since most irAEs were observed within 3 months of initiating ipilimumab (induction phase), a 3-month landmark adjustment was made for all analyses involving OS and RFS. ${ }^{8}$ The landmark analysis provides a valid method of evaluating OS and RFS by irAE status by only including cases who were alive at a 3-month landmark time regardless of irAE status.

Cox proportional hazards regression models were developed to evaluate the prognostic significance of individual irAEs adjusting for established prognostic factors, including age, gender, ECOG PS, Breslow thickness of the primary tumor, Clark level, ulceration status, mitotic rate and AJCC stage group. The categoric variables used are presented in table 1; continuous variables (age, size of primary melanoma lesion and Breslow depth) were dichotomized using the median value. Fisher's exact test was used to compare associations of categorical data.

Cox multivariate models were developed using the SAS stepwise selection process with a significance level of 0.1 for variable entry into the model and 0.1 to remain in the model. HRs were estimated and presented with $95 \%$ CIs. Two-sided $p$ values were reported for all analyses. Multiplicity was not corrected as this was a secondary analysis of irAE data. All analyses were conducted using SAS software V.9.4.

\section{RESULTS}

The characteristics of patients enrolled in E1609 and the treatment details as well as the incidence rate of irAEs were previously published. ${ }^{6}$ Patient disposition is described in the consort diagram (figure 1). Table 1 summarizes the baseline and disease characteristics of the 1034 patients randomized to the ipilimumab arms. The use of corticosteroids, immunosuppressants and hormone replacement therapy in order to manage irAEs is summarized by treatment arm and by irAE severity in table 2 . The HDI arm is included as a comparator. Non-corticosteroid immunosuppressants most commonly included infliximab, mycophenolate and cyclosporine. Hormones used to manage endocrinopathies included thyroid hormone, hydrocortisone and testosterone. There was significantly more use 
Table 1 Summary of patient baseline and disease characteristics for E1609 patients randomized to the ipilimumab arms

\begin{tabular}{|c|c|c|}
\hline Characteristic & $\begin{array}{l}\text { No. of patients } \\
(n=1034)\end{array}$ & $\%$ \\
\hline 5-year OS rate, $95 \% \mathrm{Cl}$ & - & 71 (68 to 74$)$ \\
\hline 5-year RFS rate, $95 \% \mathrm{Cl}$ & - & 48 (44 to 51$)$ \\
\hline Age, years & & - \\
\hline Median & 54 & \\
\hline Range & $18-80$ & \\
\hline \multicolumn{3}{|l|}{ Sex } \\
\hline Male & 670 & 64.8 \\
\hline Female & 364 & 35.2 \\
\hline \multicolumn{3}{|l|}{ ECOG PS } \\
\hline 0 & 865 & 83.6 \\
\hline 1 & 167 & 16.2 \\
\hline Missing & 2 & 0.2 \\
\hline Breslow depth, $\mathrm{mm}$ & & - \\
\hline Median & 2.5 & \\
\hline Range & $0-6.7$ & \\
\hline \multicolumn{3}{|l|}{ Clark level } \\
\hline I & 10 & 1 \\
\hline II & 30 & 2.9 \\
\hline III & 119 & 11.5 \\
\hline IV & 552 & 53.4 \\
\hline V & 105 & 10.2 \\
\hline Missing & 218 & 21.1 \\
\hline \multicolumn{3}{|l|}{ AJCC stage group } \\
\hline IIIB & 548 & 53 \\
\hline IIIC & 410 & 39.7 \\
\hline M1a & 56 & 5.4 \\
\hline M1b & 20 & 1.9 \\
\hline \multicolumn{3}{|l|}{ Ulceration } \\
\hline No & 405 & 39.1 \\
\hline Yes & 479 & 46.3 \\
\hline Missing & 150 & 14.6 \\
\hline \multicolumn{3}{|l|}{ Mitotic rate } \\
\hline 0 & 41 & 4.0 \\
\hline 1 & 114 & 11.0 \\
\hline 2 to 3 & 146 & 14.1 \\
\hline $4+$ & 491 & 47.5 \\
\hline Missing & 242 & 23.4 \\
\hline \multicolumn{3}{|l|}{ Treatment arm } \\
\hline Ipilimumab 3 mg/kg & 511 & 49.4 \\
\hline Ipilimumab 10 mg/kg & 523 & 50.6 \\
\hline
\end{tabular}

ECOG PS, Eastern Cooperative Oncology Group performance status; OS, overall survival; RFS, relapse-free survival.

of corticosteroids, immunosuppressants and hormone replacement therapy on the ipi10 arm compared with the ipi3 arm ( $75.9 \%$ vs $57.4 \%$; p value $<0.001)$. Furthermore, the use of 'corticosteroids or immunosuppressants' was significantly more frequent among patients experiencing grades 3-4 irAE (92\%) compared with those experiencing grades $1-2(60 \%)(\mathrm{p}<0.001)$. However, when testing the association between any use of corticosteroids and immunosuppressants for any grade irAE and RFS or OS, no significant associations were found.

We assessed the association between non-corticosteroid immunosuppressant use and outcome (RFS and OS) among cases who experienced grades 1-4 irAEs in the ipi10 and ipi3 arms. Of 896 cases with grades 1-4 irAEs, 67 cases used immunosuppressants and 829 cases did not. The 5-year RFS rates were $40.2 \%$ (95\% CI 27.7 to 52.5) for the cases who used immunosuppressants and 49.5 (95\% CI 45.8 to 53.1) for the cases who did not. Similarly, the 5 -year OS rates were $69.3 \%$ (95\% CI 56.1 to 79.3 ) for the cases who used immunosuppressants and $72.3 \%$ (95\% CI 68.9 to 75.3) for the cases who did not. Although there were trends toward better outcomes favoring cases who did not use immunosuppressants, no significant associations were found.

In investigating the association between irAEs and outcome, there was a significant association between grades 1-2 irAEs (vs no irAEs) and RFS as shown in figure 2A. The 5-year RFS rates were 52\% (95\% CI $47 \%$ to $56 \%$ ) versus $41 \%$ (95\% CI $31 \%$ to $50 \%$ ) with no irAE; $\mathrm{p}=0.006$. In testing the association between grades $1-2$ irAEs (vs no irAE) and OS, the 5-year OS rates were $75 \%$ (95\% CI $71 \%$ to $79 \%$ ) with irAEs compared with $67 \%$ (95\% CI 56\% to $75 \%$ ) with no irAE; $\mathrm{p}=0.064$ (figure $2 \mathrm{~B}$ ). Including severe irAEs in the analysis (grades 1-4), the association persisted. The 5-year RFS rates were $49 \%$ (95\% CI $45 \%$ to $52 \%$ ) with grades 1-4 irAEs compared with $41 \%(95 \% 31 \%$ to $50 \%$ ) with no irAE, as shown in online supplemental figure $\mathrm{S} 1 \mathrm{~A} ; \mathrm{p}=0.010$. The association was not significant for OS (online supplemental figure S1B). Figure 3A (RFS) and 3B (OS) show the KaplanMeier plots for patients with grades 1-2 versus grades 3-4 versus no irAEs. Patients with grades 1-2 irAEs had the best prognosis in terms of RFS and OS and those with grades 3-4 had less RFS benefits and no OS advantage over no irAE.

We investigated the contributions of the individual toxicities to the associations observed with RFS and OS, testing those with grades 1-2 irAEs versus those with no irAE in a univariate analysis (log-rank test). Rash and endocrinopathy provided the most significant associations. These were followed by neurologic irAEs, then diarrhea/colitis. Table 3 summarizes the 5-year RFS and OS rates by irAE type, testing grade 1-2 versus grade 0 .

In developing a multivariate model, univariate analyses were first carried out using the incidence of worst grade irAE, testing grades $1-2(n=525)$ or $1-4 \quad(n=525+371)$ versus no irAE $(n=115)$. The categorical prognostic variables were tested as defined in table 1 , and the continuous variables (age, Breslow depth, Clark level and mitotic rate) were dichotomized using the median value. We treated RFS and OS as outcomes separately 


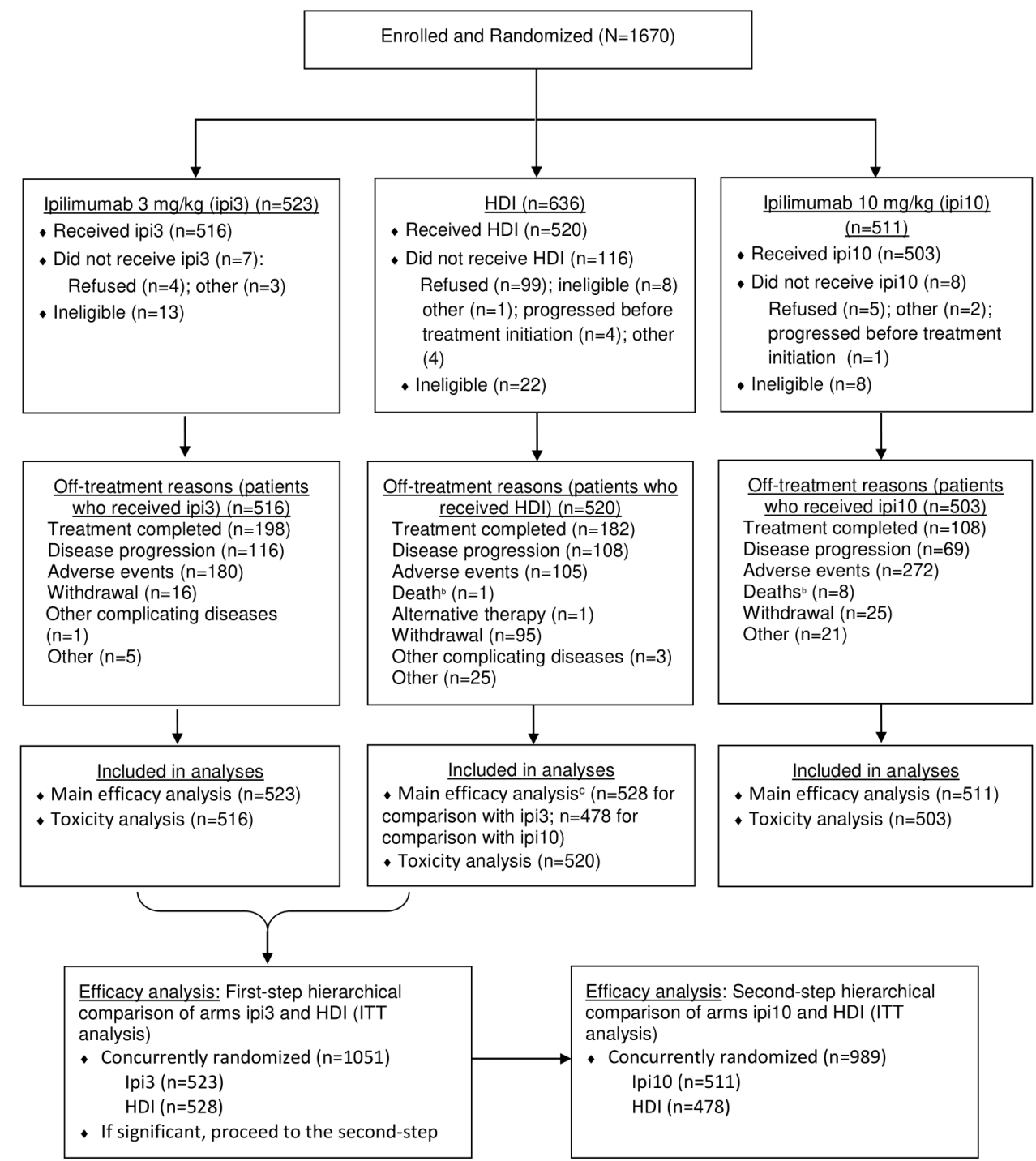

Figure 1 E1609 Consolidated Standards of Reporting Trials diagram (adult patient populationa). ${ }^{a} \mathrm{E} 1609$ included a pediatric component (ages 12-17 years) consisting of three separate cohorts randomized to the three treatment regimens and analyzed separately for safety per study protocol. Total pediatric accrual was three subjects; ${ }^{b}$ these overlap with but are not limited to treatment-related grade 5 events previously reported; ' ${ }^{c}$ concurrently randomized cases. HDI, high-dose interferon alpha-2b; Ipi3, ipilimumab 3 mg/kg; Ipi10, ipilimumab 10 mg/kg; ITT, Intent to Treat.

and combined the data from the two ipilimumab arms. Table 4 summarizes the univariate analysis outcomes. We selected any irAE grades $1-2$, any irAE grades $1-4$, rash, endocrine, endocrine plus rash and gastrointestinal (GI) as our main adverse events in this analysis because of the statistically significant associations with RFS and OS seen in the univariate analysis. Online supplemental table S5 summarizes the outcomes of the multivariate models for grades $1-2$, grades $1-4$, rash and endocrine plus rash that maintained significant associations after adjusting for other significant prognostic factors. We show our findings related to endocrine and GI irAEs below.

\section{Rash}

We compared any rash irAE versus no rash irAE; 496 patients had a grades 1-2 rash irAE and 498 no rash irAE. In the multivariate Cox models, the occurrence of grades 1-2 rash was significantly associated with RFS
( $\mathrm{p}=0.001, \mathrm{HR}=0.71 ; 95 \% \mathrm{CI} 0.57$ to 0.87 ) while adjusting for age $(p=0.013, H R=1.31)$, Breslow thickness $(p=0.036$, $\mathrm{HR}=1.29)$, Clark level $(\mathrm{p}<0.003, \mathrm{HR}=1.31)$, $\mathrm{PS}(\mathrm{p}=0.040$, $\mathrm{HR}=1.31)$ and stage group $(\mathrm{p}<0.001, \mathrm{HR}=1.36)$ (online supplemental table S5C). Similarly, grades 1-2 rash was significantly associated with $\mathrm{OS}(\mathrm{p}=0.024, \mathrm{HR}=0.72$; $95 \%$ CI 0.55 to 0.96$)$ while adjusting for age $(p=0.025$, $\mathrm{HR}=1.38)$ and Clark level $(\mathrm{p}=0.002, \mathrm{HR}=1.40)$ (online supplemental table S5D). Similar observations were found in testing grades $1-4$ rash $(\mathrm{n}=536)$ versus no rash irAE $(n=498)$ as shown in online supplemental table S5E,F.

\section{Endocrine}

We compared any endocrine irAE versus no endocrine irAE; 299 had grades 1-2 endocrine irAEs and $690 \mathrm{had}$ no endocrine irAE. In the multivariate Cox models, the occurrence of grades 1-2 endocrine irAEs was not significantly associated with RFS ( $p=0.200, H R=0.86)$ after 
Table 2 Use of corticosteroids, immunosuppressants and hormone replacement therapy by E1609 treatment arm (A) and by irAE severity among patients treated with ipilimumab (Ipi3+lpi10) (B)

\begin{tabular}{|c|c|c|c|}
\hline A & Ipi3 (n=523) & HDI ( $n=636)$ & Ipi10 (n=511) \\
\hline Corticosteroids, n (\%) & $299(57.2)$ & $66(10.4)$ & $387(75.7)$ \\
\hline Immunosuppressants*, n (\%) & $28(5.4)$ & $4(0.6)$ & $42(8.2)$ \\
\hline Corticosteroids or immunosuppressants, n (\%) & $300(57.4)$ & $68(10.7)$ & $388(75.9)$ \\
\hline B & $\begin{array}{l}\text { No irAE } \\
(n=115)\end{array}$ & irAE grades 1 and $2(n=525)$ & irAE grades 3 , and $4(n=371)$ \\
\hline Immunosuppressants*, n (\%) & $2(1.7)$ & $10(1.9)$ & $57(15.4)$ \\
\hline Hormones†, n (\%) & $4(3.5)$ & $113(21.5)$ & $119(32.1)$ \\
\hline Corticosteroids or immunosuppressants, $\mathrm{n}(\%)$ & $18(15.7)$ & $315(60.0)$ & $342(92.2)$ \\
\hline
\end{tabular}

*Infliximab, mycophenolate, cyclosporine and other.

†Thyroid hormone, hydrocortisone and testosterone.

HDI, high-dose interferon; Ipi3, ipilimumab 3 mg/kg; Ipi10, ipilimumab 10 mg/kg; irAE, immune-related adverse event.

adjusting for age $(\mathrm{p}=0.012, \mathrm{HR}=1.26)$, Breslow thickness $(\mathrm{p}=0.001, \mathrm{HR}=1.34)$, Clark level $(\mathrm{p}<0.001, \mathrm{HR}=1.36)$ and stage group $(\mathrm{p}<0.001, \mathrm{HR}=1.29)$ or OS $(\mathrm{p}=0.400$, $\mathrm{HR}=0.87)$ after adjusting for age $(\mathrm{p}=0.019, \mathrm{HR}=1.33)$, Breslow thickness $(\mathrm{p}=0.002, \mathrm{HR}=1.45)$ and Clark level $(\mathrm{p}<0.001, \mathrm{HR}=1.43)$. Similar observations were found in testing grades $1-4$ endocrine irAEs $(n=344)$ versus no endocrine irAE $(n=690)$.

\section{Endocrine plus rash}

We compared endocrine+rash $\mathrm{AE}$ versus no endocrine or rash irAE; 759 patients had either an endocrine or rash irAE or both and 176 did not. In the multivariate Cox models, the occurrence of grades 1-2 endocrine+rash irAEs was significantly associated with RFS $(p<0.001$, $\mathrm{HR}=0.66)$ after adjusting for age $(\mathrm{p}=0.006, \mathrm{HR}=1.29)$, stage group ( $<<0.001, H R=1.32)$, Breslow thickness $(\mathrm{p}=0.001$, $\mathrm{HR}=1.37)$, Clark level $(\mathrm{p}<0.001, \mathrm{HR}=1.35)$ and mitotic rate $(\mathrm{p}=0.05, \mathrm{HR}=1.05)$ and $\mathrm{OS}(\mathrm{p}=0.008, \mathrm{HR}=0.7)$ after adjusting for age $(\mathrm{p}=0.009, \mathrm{HR}=1.39)$, Breslow thickness $(\mathrm{p}=0.003, \mathrm{HR}=1.45)$ and Clark level $(\mathrm{p}=0.009, \mathrm{HR}=1.41)$ as shown in online supplemental table S5G,H. Similar observations were found in testing grades 1-4 endocrine $(\mathrm{n}=858)+$ rash irAEs versus no endocrine or rash irAE $(\mathrm{n}=176)$ as shown in online supplemental table S5I,J.

\section{Gastrointestinal}

We compared any GI irAE versus no GI irAE; 449 had a grade 1-2 GI irAE versus no GI irAE $(\mathrm{n}=507)$. In the multivariate Cox models, the occurrence of grades 1-2 GI irAEs was not significantly associated with RFS $(\mathrm{p}=0.3$, $\mathrm{HR}=0.9)$ after adjusting for age $(\mathrm{p}=0.006, \mathrm{HR}=1.29)$, stage group $(\mathrm{p}<0.001, \mathrm{HR}=1.28)$, Breslow thickness $(\mathrm{p}<0.001$, $\mathrm{HR}=1.40)$, Clark level $(\mathrm{p}<0.001, \mathrm{HR}=1.34)$ and mitotic rate $(\mathrm{p}=0.02, \mathrm{HR}=1.06)$ or $\mathrm{OS}(\mathrm{p}=0.48, \mathrm{HR}=0.9)$ after adjusting for age $(\mathrm{p}=0.009, \mathrm{HR}=1.38)$, Breslow thickness $(\mathrm{p}<0.001, \mathrm{HR}=1.56)$ and Clark level $(\mathrm{p}<0.001, \mathrm{HR}=1.45)$. Similar observations were found in testing grades 1-4 GI irAEs $(n=527)$ versus no GI irAE $(n=507)$.

\section{DISCUSSION}

CTLA4 blockade with ipilimumab as tested in E1609 for adjuvant therapy of high-risk melanoma was associated with significant irAEs that appear to be related to the underlying immune mechanism of action of this antiCTLA4 antibody. As immune checkpoints play a pivotal role in regulating tolerance to self-antigens, blockade can result in autoimmune damage of various organ systems, leading to irAEs. There was a significant association between high-grade irAEs (3-4) and the use of corticosteroids and immunosuppressants. Among patients with grades 3-4 diarrhea/colitis, $37.2 \%$ required additional use of immunosuppressants such as infliximab. Therefore, we decided to investigate the differential impact of varying irAE grades on patient outcome. In testing the association between irAEs and clinical outcome, we first evaluated the lower grade AEs, grades 1-2 and whether significant associations were seen in relation to RFS and OS. We later added the severe adverse events to the analysis (grades 1-4) and noted that the associations persisted, although the survival benefits appeared to erode. As presented in figure 3A,B, patients with grades 1-2 irAEs had the best prognosis in terms of RFS and OS and those with grades 3-4 had less RFS benefit and no OS advantage over no irAE. These observations raise questions about the potential negative impact on clinical outcome of high-dose corticosteroids and other immunosuppressants that were primarily initiated during the ipilimumab induction phase in response to grades $3-4$ toxicities as required by the study protocol and toxicity management guidelines. This is compared with primarily symptomatic management of grade 1 irAEs and the use of low to moderate dose corticosteroids to manage grade 2 irAEs. Other reports have suggested an adverse effect of high dose corticosteroid exposure (primarily at baseline) on survival in patients with melanoma and other malignancies treated with ICIs. ${ }^{9-11}$ These data warrant further investigation and support a review and potential revisions 

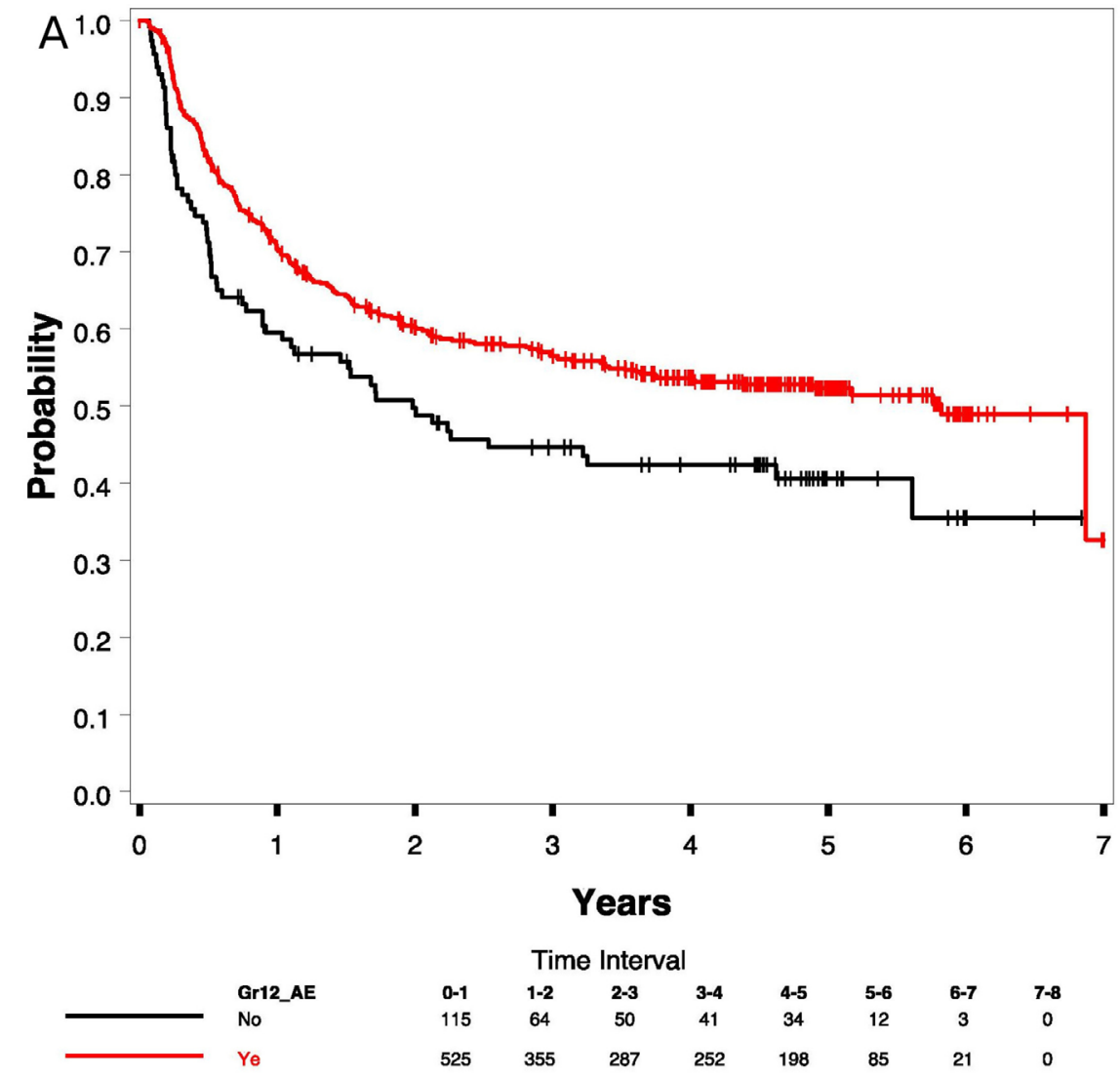

(\# at risk)
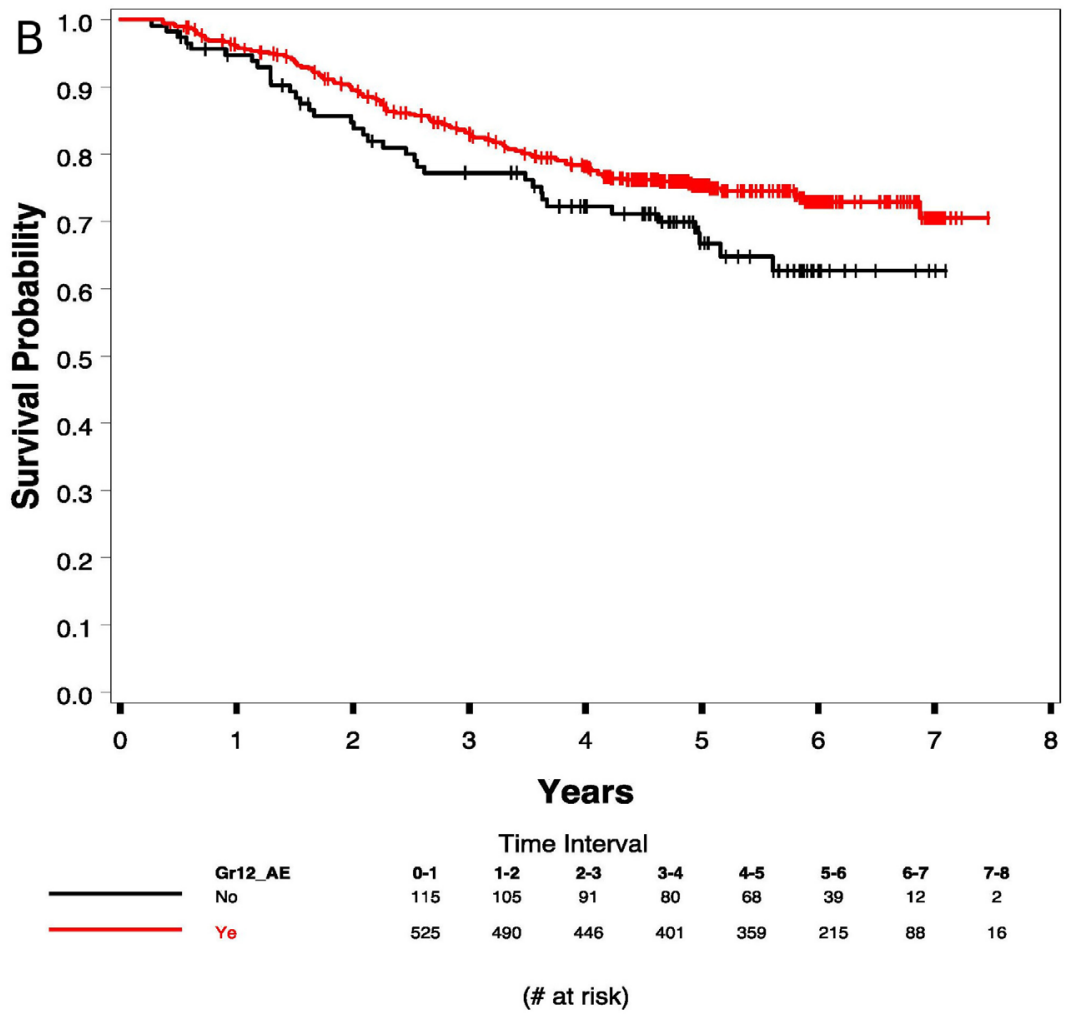

Figure 2 Kaplan-Meier plots of relapse-free survival (RFS) (A: 5-year RFS 52\% (95\% Cl 47\% to 56\%) versus 41\% (95\% Cl $31 \%$ to $50 \%$ ); $p=0.006$ ) and overall survival (OS) (B: 5-year OS 75\% (95\% Cl $71 \%$ to $79 \%$ ) vs $67 \%$ (95\% Cl 56\% to $75 \%$ ); $\mathrm{p}=0.064$ ) with grade 1-2 irAEs versus no irAE, respectively. irAE, immune-related adverse event. 

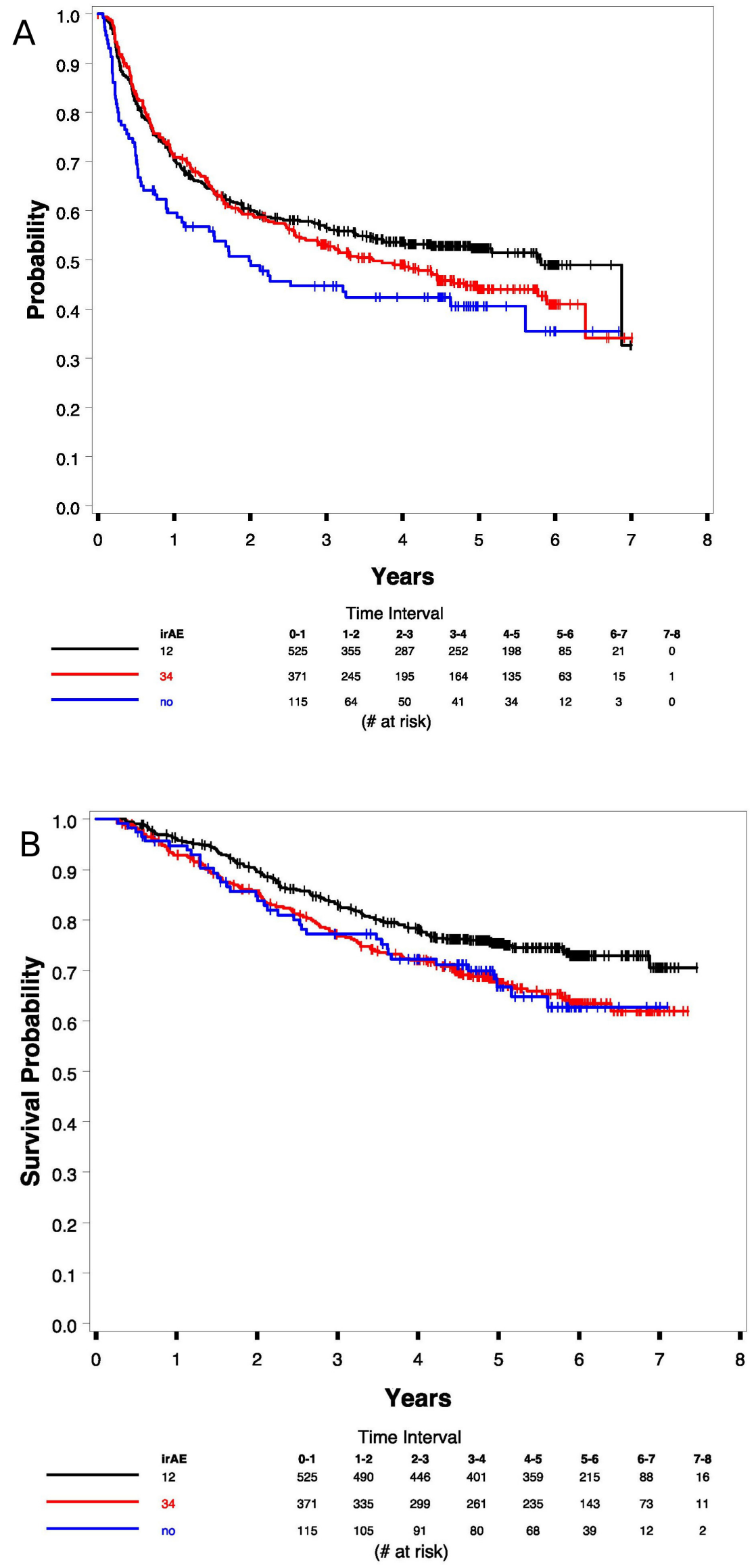

Figure 3 Kaplan-Meier plots of relapse-free survival (A) and overall survival (B) with grades 1-2 versuss grades 3-4 veruss no irAE. irAE, immune-related adverse event. 
Table 3 Five-year RFS and OS rates for patients with individual irAEs and a highest CTCAE grades of 1-2 versus grade 0

\begin{tabular}{|c|c|c|c|c|c|c|}
\hline & \multicolumn{3}{|c|}{ 5-year RFS, \% (95\% Cl) } & \multicolumn{3}{|c|}{ 5-year OS, \% (95\% Cl) } \\
\hline & Grades1-2 & No AE & P value* & Grades 1-2 & No AE & P value* \\
\hline Rash & 52 (47 to 57 ) & 45 (40 to 50 ) & 0.002 & 75 (71 to 79$)$ & 68 (65 to 72$)$ & 0.004 \\
\hline Endocrine and rash & 54 (49 to 53 ) & 41 (36 to 47$)$ & $<0.001$ & 77 (73 to 80$)$ & 68 (62 to 73$)$ & $<0.001$ \\
\hline Diarrhea/colitis & 51 (46 to 57 ) & 48 (45 to 51$)$ & 0.094 & 72 (68 to 77$)$ & $71(66$ to 75$)$ & 0.343 \\
\hline
\end{tabular}

*Based on the log-rank test.

CTCAE, Common Terminology for Adverse Events

; irAE, immune-related adverse event; OS, overall survival; RFS, relapse-free survival.

of current irAE management guidelines. One could also envision the investigation of adaptive strategies that may judiciously minimize the use of corticosteroids and other immunosuppressants by dose and duration.

In investigating the contributions of the individual toxicities, rash followed by endocrinopathy provided the most significant associations. Similar but weaker associations were seen for neurologic irAEs followed by diarrhea/ colitis. The associations persisted through multivariate modeling analyses adjusted for established prognostic factors in this prospectively conducted randomized controlled trial. While one retrospective analysis of metastatic melanoma patients treated with ipilimumab showed no association between irAEs and clinical outcome, ${ }^{12}$ another retrospective study of 139 patients with metastatic melanoma who received ipilimumab with or without a peptide vaccine reported that the development

Table 4 Cox univariate analysis outcomes by toxicity type for irAE CTCAE grades 1-2 or grade 1-4 versus grade 0

\begin{tabular}{|c|c|c|}
\hline \multirow[b]{2}{*}{ Toxicity type } & \multicolumn{2}{|c|}{ HR (95\% Cl) } \\
\hline & RFS & os \\
\hline Any irAE grades $1-2$ & $\begin{array}{l}0.60(0.52 \text { to } 0.90) \\
p=0.006\end{array}$ & $\begin{array}{l}0.71(0.49 \text { to } 1.02), \\
p=0.065\end{array}$ \\
\hline Any irAE grades $1-4$ & $\begin{array}{l}0.71 \text { ( } 0.55 \text { to } 0.93) \\
p=0.011\end{array}$ & $\begin{array}{l}0.82(0.58 \text { to } 1.17) \\
p=0.270\end{array}$ \\
\hline Rash grades 1-2 & $\begin{array}{l}0.75(0.62 \text { to } 0.90) \\
p=0.002\end{array}$ & $\begin{array}{l}0.70(0.55 \text { to } 0.89) \\
p=0.004\end{array}$ \\
\hline Rash grades $1-4$ & $\begin{array}{l}0.77(0.65 \text { to } 0.92) \\
p=0.004\end{array}$ & $\begin{array}{l}0.74(0.59 \text { to } 0.94), \\
p=0.012\end{array}$ \\
\hline Endo grades 1-2 & $\begin{array}{l}0.75(0.61 \text { to } 0.92) \\
p=0.005\end{array}$ & $\begin{array}{l}0.76(0.58 \text { to } 0.99) \\
p=0.045\end{array}$ \\
\hline Endo grades $1-4$ & $\begin{array}{l}0.79(0.65 \text { to } 0.97) \\
p=0.015\end{array}$ & $\begin{array}{l}0.84 \text { (0.65 to } 1.08), \\
p=0.168\end{array}$ \\
\hline $\begin{array}{l}\text { Endo and rash } \\
\text { grades 1-2 }\end{array}$ & $\begin{array}{l}0.65(0.53 \text { to } 0.78) \\
p<0.001\end{array}$ & $\begin{array}{l}0.63(0.48 \text { to } 0.81) \\
p<0.001\end{array}$ \\
\hline $\begin{array}{l}\text { Endo and rash } \\
\text { grades } 1-4\end{array}$ & $\begin{array}{l}0.73(0.57 \text { to } 0.92) \\
p=0.009\end{array}$ & $\begin{array}{l}0.73(0.57 \text { to } 0.92) \\
p<0.001\end{array}$ \\
\hline Gl grades 1-2 & $\begin{array}{l}0.87(.72 \text { to } 1.05) \\
p=0.137\end{array}$ & $\begin{array}{l}0.88(0.69 \text { to } 1.13) \\
p=0.305\end{array}$ \\
\hline Gl grades 1-4 & $\begin{array}{l}0.88(0.74 \text { to } 1.06) \\
p=0.171\end{array}$ & $\begin{array}{l}0.94(0.74 \text { to } 1.18) \\
p=0.590\end{array}$ \\
\hline
\end{tabular}

irAE, immune-related adverse event; OS, overall survival; RFS,

relapse-free survival. of irAEs was significantly associated with the likelihood of response. ${ }^{13}$ In terms of PD1 blockade, a pooled analysis of patients with metastatic melanoma treated with nivolumab reported significantly higher response rates among patients developing irAEs. Separately, the development of cutaneous adverse events was associated with significantly longer progression-free survival in patients treated with pembrolizumab. ${ }^{14}$ In the melanoma adjuvant setting, an association between irAEs and improved RFS was reported among stage III patients treated with pembrolizumab. ${ }^{15}$ We also had previously reported an association between the development of autoimmunity and improved outcome for patients treated tremelimumab and interferon- $\alpha .{ }^{16}$ Separate from ICIs, we and others reported that the development of autoimmunity is a favorable prognostic factor following cytokine therapy with interferon- $\alpha$ and interleukin-2. ${ }^{17}$ is

Predictors of irAE risk and understanding the underlying mechanisms are a major gap and need to be thoroughly investigated. Important clues to understanding irAEs come from autoimmune diseases. Many irAEs have substantial similarities to known autoimmune disorders. For example, colitis appears histologically similar to inflammatory bowel disease (IBD), characterized by a lymphocytic and neutrophilic infiltration, ${ }^{19}{ }^{20}$ and has a similar gene expression signature in the infiltrate of immunotherapy-related colitis and Crohn's disease. ${ }^{21}$ Similar to IBD, immune-related colitis responds to steroids and Interferon Alfa (TNF- $\alpha$ ) blockade. ${ }^{1}$ Genetic variation at the human leukocyte antigen (HLA) locus is associated with a substantially increased risk of the development of autoimmune disorders. Type 1 diabetes is strongly associated with particular HLA haplotypes with ORs up to 10 for some of the haplotypes. ${ }^{22}$ Large effects also have been noted for autoimmune hepatitis (threefold), ${ }^{23}$ inflammatory bowel diseases (3.7-fold) ${ }^{24}$ and thyroiditis (fourfold) ${ }^{25}$ which are similar in nature to those observed here for ICI irAEs. Particularly intriguing, genomic variation at the CTLA4 locus is associated with type 1 diabetes, rheumatoid arthritis, Graves' disease, alopecia areata and celiac disease. ${ }^{26}$ Furthermore, variants near the PD-1 locus have been associated in genome-wide association studies for Crohn's disease. ${ }^{27}$ These data support the hypothesis that inherited variations contribute to irAE susceptibility, which may have substantial overlap with the susceptibility 
to autoimmune diseases apart from ICI therapy. They support testing the association of inherited genetic variation and irAEs in patients treated with ICIs. Furthermore, they support investigating the association of inherited genetic variants related to autoimmunity and irAEs with OS and RFS. Our group and others have ongoing efforts in this setting.

While the exact pathophysiology of immune toxicity with ICIs is not clear, many host inflammatory and immunoregulatory cells appear to be involved. ${ }^{19}{ }^{21}$ Biopsies of the GI tract from patients with colitis following treatment with ipilimumab showed neutrophilic and lymphocytic inflammation with evidence of increased CD8+ T cell infiltration within the crypt epithelium and increased CD4+ $\mathrm{T}$ cells in the lamina propria. ${ }^{19}$ We had reported a role for Th17 cells and IL17 in relation to the development of immune-mediated colitis in patients treated with ipilimumab. ${ }^{28}$ In a randomized trial by ECOG-ACRIN testing ipilimumab with or without GM-CSF, there were significantly fewer colitis and pneumonitis irAEs observed with the addition of GM-CSF, suggesting a role for GM-SCF in maintaining GI and pulmonary immunoregulatory homeostasis. ${ }^{29}$ The ongoing ECOG-ACRIN EA6141 trial is currently testing the role of GM-CSF in combination with ipilimumab and nivolumab.

\section{CONCLUSIONS}

Adjuvant therapy with ipilimumab is associated with a risk for significant irAEs that appears to be related to the immune mechanism of action of this agent. Patients experiencing lower grade irAEs (grades 1-2) derive the most benefit, but we found no significant evidence supporting a negative impact of high-dose corticosteroids and immunosuppressants used to manage grades 3-4 irAEs. Predictors of the risk for irAEs and a fundamental understanding the underlying mechanisms of these irAEs remain major gaps and are currently being actively investigated.

\section{Author affiliations}

${ }^{1}$ Departments of Cutaneous Oncology and Immunology, H. Lee Moffitt Cancer Center and Research Center Inc, Tampa, Florida, USA

${ }^{2}$ Dana-Farber Cancer Institute and Harvard Medical School, Boston, Massachusetts, USA

${ }^{3}$ Dana-Farber Cancer Institute, Boston, Massachusetts, USA

${ }^{4}$ Greater Baltimore Medical Center, Baltimore, Maryland, USA

${ }^{5}$ The Angeles Clinic \& Research Institute, A Cedars Sinai Affiliate, Los Angeles,

California, USA

${ }^{6}$ Department of Medicine, University of Arkansas for Medical Sciences (UAMS), Little Rock, Arkansas, USA

${ }^{7}$ Robert H. Lurie Comprehensive Cancer Center of Northwestern University, Chicago, Illinois, USA

${ }^{8}$ Department of Medicine, Yale University, New Haven, Connecticut, USA

${ }^{9}$ Case Western Reserve University, Cleveland, Ohio, USA

${ }^{10}$ Massachusetts General Hospital, Boston, MA, USA

${ }^{11}$ Ohio State University, Columbus, OH, USA

${ }^{12}$ Sutter-California Pacific Medical Center, San Francisco, California, USA

${ }^{13}$ University of North Carolina at Chapel Hill, Chapel Hill, North Carolina, USA

${ }^{14}$ University of Wisconsin, Madison, Wisconsin, USA

${ }^{15}$ Wayne State University and Karmanos Cancer Institute, Detroit, Michigan, USA

${ }^{16}$ Odette Cancer Center, Toronta, Ontario, Canada

${ }^{17}$ National Cancer Institute, Rockville, MD, USA
${ }^{18}$ UPMC Hillman Cancer Center, Pittsburgh, Pennsylvania, USA

Acknowledgements We would like to thank the patients and their families and the investigators who participated in the E1609 study.

Contributors Conception and design: AT, SJL and NK. Provision of study material or patients: AT, JMK, FSH, VKS, GC, OH, LFH, JAS, HK, ZE, HBK, DPL, KK, DRM, CBL, MRA, LF and TMP. Collection and assembly of data: AT, JMK, SJL, FSH, VKS, GC, OH, LFH, JAS, HK, ZE, HK, DPL, KK, DRM, CBL, MRA, LF and TMP. Data analysis: AT, SL and NK. Data interpretation: AT, SL, NK, FSH, JMK, VKS, GC, OH, LFH, JAS, HK, ZE, HK, DPL, KK, DRM, CBL, MRA, LF and TMP. Manuscript writing: all authors. Final approval of manuscript: all authors. Accountable for all aspects of the work: all authors.

Funding This study was coordinated by the ECOG-ACRIN Cancer Research Group (Peter J O'Dwyer, MD, and Mitchell D Schnall, MD, PhD, Group Co-Chairs) and supported by the National Cancer Institute of the National Institutes of Health under the following award numbers: U10CA180820, U10180794, U10CA180821, U10CA180863, U10CA180888, UG1CA189859, UG1CA233163, UG1CA233180, UG1CA233184, UG1CA233196, UG1CA233234, UG1CA233237, UG1CA233270, UG1CA233331, UG1CA233373, UG1CA233320, UG1CA233337, Canadian Cancer Society \#704 970. This study was also supported by Bristol-Myers Squibb.

Disclaimer The content is solely the responsibility of the authors and does not necessarily represent the official views of the National Institutes of Health. Mention of trade names, commercial products or organizations does not imply endorsement by the US government.

Competing interests AT reports grants from National Cancer Institute, National Institute of Health, ECOGACRIN, grants from Bristol Myers Squibb, during the conduct of the study; grants from Bristol Myers Squibb, personal fees from Bristol Myers Squibb, grants from Merck, personal fees from Merck, personal fees from Novartis, personal fees from Genentech-Roche, personal fees from Array Biopharma, grants from Incyte, personal fees from Incyte, personal fees from NEWLINK Genetics, personal fees from HUYA, personal fees from BioNTech, grants from Prometheus, personal fees from Immunocore, grants from Greenpeptide, grants from Amgen, outside the submitted work. SJL has nothing to disclose. FSH reports clinical trial support from Eastern Cooperative Oncology Group, during the conduct of the study; grants, personal fees and other from Bristol Myers Squibb, personal fees from Merck, personal fees from EMD Serono, grants and personal fees from Novartis, personal fees from Takeda, personal fees from Surface, personal fees from Genentech/Roche, personal fees from Compass Therapeutics, personal fees from Apricity, personal fees from Bayer, personal fees from Aduro, personal fees from Partners Therapeutics, personal fees from Sanofi, personal fees from Pfizer, personal fees from Pionyr, from 7 Hills Pharma, personal fees from Verastem, other from Torque, personal fees from Rheos, outside the submitted work; in addition, FSH has a patent Methods for Treating MICA-Related Disorders (\#20100111973) with royalties paid, a patent Tumor antigens and Uses Thereof (\#7250291) issued, a patent Angiopoiten-2 Biomarkers Predictive of Antiimmunecheckpoint response (\#20170248603) pending, a patent Compositions and Methods for Identification, Assessment, Prevention, and Treatment of Melanoma using PD-L1lsoforms (\#20160340407) pending, a patent Therapeutic peptides (\#20160046716) pending, a patent Therapeutic Peptides (\#20140004112) pending, a patent Therapeutic Peptides (\#20170022275) pending, a patent Therapeutic Peptides (\#20170008962) pending, a patent Therapeutic Peptides Patent number: 9402905 issued, and a patent Methods of Using Pembrolizumab and Trebananib pending. GC has nothing to disclose. $\mathrm{OH}$ reports personal fees from BMS, during the conduct of the study; and Contracted Research for Institution: Amgen, Arcus, Astellas, AstraZeneca, BMS, Celldex, CytomX,Genentech, GSK, Immunocore, Incyte, lovance, Merck, Merck Serono, Medlmmune,Nextcure, Novartis, Parker, Pfizer, Polynoma, Regeneron and Roche. LFH has nothing to disclose. JAS reports personal fees from BMS, personal fees from Array, personal fees from Curis, personal fees from MSD, personal fees from Roche, outside the submitted work. HMK reports grants from Merck, grants from Bristol Myers Squibb, grants from Apexigen, personal fees from Regeneron, personal fees from Alexion, personal fees from Prometheus, personal fees from Corvus, personal fees from Nektar, personal feesfrom Biodesix, personal fees from Roche-Genentech, personal fees from Pfizer, personal fees from lovance, personal fees from Immunocore, personal fees from Celldex, outside the submitted work. HBK reports grants from BMS, during the conduct of the study; other from BMS, outside the submitted work; and he is currently employed by BMS. DPL has nothing to disclose. KLK reports clinical trial support from Immunocore, clinical trial support from Medspace, clinical trial support from Regeneron, clinical trial support from Novartis, grants from BMS, clinical trial support from GlaxoSmithKline, clinical trial support from Karyopharm, grants from 
Merck outside the submitted work. DRM reports stock ownership from Nektar, Inc and BMS outside of submitted work. CL reports personal fees from Delcath, Inc, outside the submitted work. MRA reports grants from NIH, during the conduct of the study; research collaboration with Bristol Myers Squibb and Apeiron Biologics, outside the submitted work. LF has nothing to disclose. ZE reports advisory role with Array, Regeneron, and Compugen and grants from Novartis, outside the submitted work. TMP reports personal fees from BMS, personal fees from Merck, during the conduct of the study; grants and personal fees from Novartis, grants from Roche, personal fees from Sanofi Genzyme, outside the submitted work. HS has nothing to disclose. VKS reports personal fees from Merck, Bristol Myers Squibb, Novartis, Array, Polynoma, Pfizer and Regeneron, outside the submitted work. JMK reports grants and personal fees from BMS, grants and personal fees from Immunocore, personal fees from Novartis, personal fees from lovance, grants from Merck, personal fees from Amgen, grants from Checkmate, personal fees from Elsevier, outside the submitted work.

\section{Patient consent for publication Not required.}

Ethics approval The study protocol was approved by the institutional review board of each participating institution and conducted in accordance with Good Clinical Practice guidelines as defined by the International Conference on Harmonization. All patients provided an Institutional Review Board (IRB)-approved written informed consent. This study was monitored by the ECOG-ACRIN Data Safety Monitoring Committee and the National Cancer Institute $(\mathrm{NCl})$.

Provenance and peer review Not commissioned; externally peer reviewed.

Data availability statement Data are available in a public, open access repository. The data sets generated, analyzed and reported in the present paper will be made available in theNational Clinical Trials Network/ NCl Community Oncology Research Program (NCTN/NCORP) Data Archive (https://nctn-data-archive.nci.nih.gov).

Supplemental material This content has been supplied by the author(s). It has not been vetted by BMJ Publishing Group Limited (BMJ) and may not have been peer-reviewed. Any opinions or recommendations discussed are solely those of the author(s) and are not endorsed by BMJ. BMJ disclaims all liability and responsibility arising from any reliance placed on the content. Where the content includes any translated material, BMJ does not warrant the accuracy and reliability of the translations (including but not limited to local regulations, clinical guidelines, terminology, drug names and drug dosages), and is not responsible for any error and/or omissions arising from translation and adaptation or otherwise.

Open access This is an open access article distributed in accordance with the Creative Commons Attribution Non Commercial (CC BY-NC 4.0) license, which permits others to distribute, remix, adapt, build upon this work non-commercially, and license their derivative works on different terms, provided the original work is properly cited, appropriate credit is given, any changes made indicated, and the use is non-commercial. See http://creativecommons.org/licenses/by-nc/4.0/.

\section{ORCID iD}

Ahmad A Tarhini http://orcid.org/0000-0002-3193-9702

\section{REFERENCES}

1 Tarhini A. Immune-Mediated adverse events associated with ipilimumab CTLA-4 blockade therapy: the underlying mechanisms and clinical management. Scientifica 2013;2013:2013.

2 Boutros C, Tarhini A, Routier E, et al. Safety profiles of anti-CTLA-4 and anti-PD-1 antibodies alone and in combination. Nat Rev Clin Oncol 2016;13:473-86.

3 Wolchok JD, Chiarion-Sileni V, Gonzalez R, et al. Overall survival with combined nivolumab and ipilimumab in advanced melanoma. $N$ Engl J Med 2017;377:1345-56.

4 Ascierto PA, Del Vecchio M, Robert C, et al. Ipilimumab 10 mg/ $\mathrm{kg}$ versus ipilimumab $3 \mathrm{mg} / \mathrm{kg}$ in patients with unresectable or metastatic melanoma: a randomised, double-blind, multicentre phase 3 trial. Lancet Oncol 2017;18:611-22.

5 Tarhini AA, Lee SJ, Kang N, et al. Immune adverse events (irAEs) with adjuvant ipilimumab in melanoma, use of hormone replacement and immunosuppressants, and association with outcome: E1609 study analysis. JCO 2020;38:60.

6 Tarhini AA, Lee SJ, Hodi FS, et al. Phase III study of adjuvant ipilimumab (3 or $10 \mathrm{mg} / \mathrm{kg}$ ) versus high-dose interferon alfa-2b for resected high-risk melanoma: North American intergroup E1609. J Clin Oncol 2020;38:567-75.
7 Kaplan EL, Meier P. Nonparametric estimation from incomplete observations. J Am Stat Assoc 1958;53:457-81.

8 Anderson JR, Cain KC, Gelber RD. Analysis of survival by tumor response. J Clin Oncol 1983;1:710-9.

9 Arbour KC, Mezquita L, Long N, et al. Impact of baseline steroids on efficacy of programmed cell death-1 and programmed Death-Ligand 1 blockade in patients with non-small-cell lung cancer. $J$ Clin Oncol 2018;36:2872-8.

10 Faje AT, Lawrence D, Flaherty K, et al. High-dose glucocorticoids for the treatment of ipilimumab-induced hypophysitis is associated with reduced survival in patients with melanoma. Cancer 2018:124:3706-14.

11 Ricciuti B, Dahlberg SE, Adeni A, et al. Immune checkpoint inhibitor outcomes for patients with non-small-cell lung cancer receiving baseline corticosteroids for palliative versus Nonpalliative indications. $J$ Clin Oncol 2019;37:1927-34.

12 Horvat TZ, Adel NG, Dang T-O, et al. Immune-related adverse events, need for systemic immunosuppression, and effects on survival and time to treatment failure in patients with melanoma treated with ipilimumab at Memorial Sloan Kettering cancer center. J Clin Oncol 2015;33:3193-8.

13 Downey SG, Klapper JA, Smith FO, et al. Prognostic factors related to clinical response in patients with metastatic melanoma treated by CTL-associated antigen-4 blockade. Clin Cancer Res 2007;13:6681-8.

14 Sanlorenzo M, Vujic I, Daud A, et al. Pembrolizumab cutaneous adverse events and their association with disease progression. JAMA Dermatol 2015;151:1206-12.

15 Eggermont AMM, Kicinski M, Blank CU, et al. Association between immune-related adverse events and recurrence-free survival among patients with stage III melanoma randomized to receive pembrolizumab or placebo: a secondary analysis of a randomized clinical trial. JAMA Oncol 2020;6:519.

16 Tarhini AA, Cherian J, Moschos SJ, et al. Safety and efficacy of combination immunotherapy with interferon alfa-2b and tremelimumab in patients with stage IV melanoma. $J$ Clin Oncol 2012;30:322-8.

17 Gogas H, loannovich J, Dafni U, et al. Prognostic significance of autoimmunity during treatment of melanoma with interferon. $N$ Engl $J$ Med 2006;354:709-18.

18 Franzke A, Peest D, Probst-Kepper M, et al. Autoimmunity resulting from cytokine treatment predicts long-term survival in patients with metastatic renal cell cancer. J Clin Oncol 1999;17:529-33.

19 Beck KE, Blansfield JA, Tran KQ, et al. Enterocolitis in patients with cancer after antibody blockade of cytotoxic T-lymphocyte-associated antigen 4. J Clin Oncol 2006;24:2283-9.

20 Wang C, Armasu SM, Kalli KR, et al. Pooled clustering of high-grade serous ovarian cancer gene expression leads to novel consensus subtypes associated with survival and surgical outcomes. Clin Cancer Res 2017;23:4077-85.

21 Balko JM, Al-Rohil RN, Wang DY, et al. Molecular characterization of immune-related severe adverse events (irSAE). JCO 2017;35:3076.

22 Erlich H, Valdes AM, Noble J, et al. Hla DR-DQ haplotypes and genotypes and type 1 diabetes risk: analysis of the type 1 diabetes genetics Consortium families. Diabetes 2008;57:1084-92.

23 de Boer YS, van Gerven NMF, Zwiers A, et al. Genome-wide association study identifies variants associated with autoimmune hepatitis type 1. Gastroenterology 2014;147:443-52.

24 Stokkers PC, Reitsma PH, Tytgat GN, et al. Hla-Dr and -DQ phenotypes in inflammatory bowel disease: a meta-analysis. Gut 1999;45:395-401.

25 Fingerlin TE, Zhang W, Yang IV, et al. Genome-wide imputation study identifies novel HLA locus for pulmonary fibrosis and potential role for auto-immunity in fibrotic idiopathic interstitial pneumonia. BMC Genet 2016;17:74

26 Zenewicz LA, Abraham C, Flavell RA, et al. Unraveling the genetics of autoimmunity. Cell 2010;140:791-7.

27 Liu JZ, van Sommeren S, Huang $\mathrm{H}$, et al. Association analyses identify 38 susceptibility loci for inflammatory bowel disease and highlight shared genetic risk across populations. Nat Genet 2015;47:979-86.

28 Tarhini AA, Zahoor $\mathrm{H}$, Lin Y, et al. Baseline circulating $\mathrm{IL}-17$ predicts toxicity while TGF- $\beta 1$ and IL-10 are prognostic of relapse in ipilimumab neoadjuvant therapy of melanoma. J Immunother Cancer 2015;3:39.

29 Hodi FS, Lee S, McDermott DF, et al. Ipilimumab plus sargramostim vs ipilimumab alone for treatment of metastatic melanoma: a randomized clinical trial. JAMA 2014;312:1744-53. 